\title{
ATTAINABLE SETS OF QUASICONCAVE MARKETS
}

\author{
ROBERT JAMES WEBER ${ }^{1}$
}

\begin{abstract}
The union of any finite collection of corners is the attainable set of a market with continuous, monotone increasing, quasiconcave utility functions. It follows that the attainable sets of such markets are dense in the collection of attainable sets of markets with utility functions restricted only to being upper-semicontinuous and lower-bounded.
\end{abstract}

One approach to the study of an economic market is to construct a cooperative $n$-person game from the market [9], [10]. Game-theoretic analysis then yields results which can be interpreted in the context of the original market. It is, therefore, of interest to characterize those games which can arise from markets of various kinds. Shapley and Shubik [10] considered markets in which utility is linearly transferable between traders. They showed that the corresponding (side-payment) games are precisely those which are totally balanced. Similar results are known for markets in which all traders have concave utility functions [3], [4], [8]. The situation for more general markets is less settled.

A natural starting point is to determine which sets of possible outcomes are associated with markets in which all traders have well-behaved utility functions. It is our purpose to investigate the character of these sets when the utility functions are restricted to being continuous, monotone increasing, and quasiconcave. We will find that these sets are dense in the collection of sets associated with markets in which the traders' utility functions are much less well behaved.

We deal with a market consisting of a set of traders $N=\{1,2, \ldots, n\}$, and an $m$-dimensional commodity space $I^{m}=\left\{\left(y_{1}, \ldots, y_{m}\right): 0 \leqslant y_{i} \leqslant 1\right\}$. (Although specification of a market usually involves the allocation of initial holdings to the traders, for our purposes we need only treat the simpler situation in which the commodities are collectively possessed and may be arbitrarily distributed.) For any collection $\left\{u_{i}\right\}_{i=1}^{n}$ of utility functions of the traders, the attainable set of the market is

$$
\mathbb{Q}\left(u_{1}, \ldots, u_{n}\right)=\left\{x \in R^{n}: x \leqslant\left(u_{1}\left(y^{1}\right), \ldots, u_{n}\left(y^{n}\right)\right)\right.
$$

$$
\text { where each } \left.y^{i} \in I^{m} \text { and } \Sigma y^{i}=(1, \ldots, 1)\right\} \text {. }
$$

Received by the editors September 26, 1975.

AMS (MOS) subject classifications (1970). Primary 90A15; Secondary 90D12.

Key words and phrases. Market games, quasiconcave utility functions, attainable sets, gametype sets, Pareto sets.

${ }^{1}$ The author is indebted to Professor Louis Billera and Mr. J. Scott Provan, of Cornell University, for their perceptive comments on several points. 
This is the set of all utility outcomes which can be achieved by some distribution of the available commodities among the traders. A set $X$ in $R^{n}$ is the comprehensive hull of another set $Y$ if $X=\left\{x \in R^{n}: x \leqslant y\right.$ for some $y \in Y\} . X$ is compactly generated if $X$ is the comprehensive hull of a compact set. A corner in $R^{n}$ is the comprehensive hull of a single point.

It has been shown by Billera and Bixby [2] that when all of the utility functions are continuous and concave, the corresponding attainable set is convex and compactly generated. They also proved a converse, that every convex, compactly generated set is the attainable set of a market with continuous, concave utility functions which are monotone increasing. Greatly weakening the restrictions on the utility functions we get the following result, the proof of which is straightforward.

THEOREM 1. If the utility functions $u_{1}, \ldots, u_{n}$ are all upper-semicontinuous and lower-bounded, then the attainable set $\mathbb{Q}\left(u_{1}, \ldots, u_{n}\right)$ is compactly generated.

The condition of lower-boundedness cannot be eliminated. Consider a market with three traders and two commodities. Define the traders' utility functions by

$$
\begin{aligned}
& u_{1}(a, b)= \begin{cases}a & \text { if } b=0 \\
0 & \text { otherwise }\end{cases} \\
& u_{2}(a, b)= \begin{cases}a & \text { if } a=b, \\
0 & \text { otherwise }\end{cases} \\
& u_{3}(a, b)= \begin{cases}-1 /(1-b) & \text { if } b<1, \\
0 & \text { otherwise. }\end{cases}
\end{aligned}
$$

These functions are clearly upper-semicontinuous. However, for every $0<\varepsilon<1$, the outcome $(1-\varepsilon, \varepsilon,-1 / \varepsilon)=\left(u_{1}(1-\varepsilon, 0), u_{2}(\varepsilon, \varepsilon), u_{3}(0,1-\varepsilon)\right)$ is in every set which has $\mathbb{Q}\left(u_{1}, u_{2}, u_{3}\right)$ as its comprehensive hull.

We shall provide a partial converse to Theorem 1 by finding a collection of attainable sets, dense in the collection of all compactly generated sets, in which each set arises from a collection of continuous, monotone increasing, quasiconcave utility functions. (Recall that a function $u$ is quasiconcave if all of its level sets $\{x: u(x) \geqslant \alpha\}$ are convex.)

TheOrem 2. Let $A$ be the union of any finite collection of corners. Then $A$ is the attainable set of a market in which all of the utility functions are continuous, monotone increasing, and quasiconcave.

We prove this theorem by constructing the desired utility functions in several stages. First, functions will be found which yield an attainable set which is the union of $A$ with at most one extraneous corner. These functions will then be modified, first to make them monotone increasing and quasiconcave, and then to make them continuous. These modifications will not change the original attainable set. Finally, the extraneous corner introduced in the 
first stage will be eliminated, and we will at last have a collection of utility functions which yields the attainable set $A$.

Let $G$ be the finite set of corner points which generates $A$. Without loss of generality, we assume $A$ to have been so affinely scaled that $G$ lies in the unit $n$-cube. Let $0=x_{0}=x_{1}<x_{2}<\ldots<x_{p}=1$ be a sequence containing all of the values taken by any of the first $(n-1)$ components of points in $G$. Select any $0<b<1 /(n-1)$. In what follows, we work with a space of $(n-1)$ commodities. A $b$-point is an $(n-1)$-vector $\left(b^{k_{1}}, \ldots, b^{k_{n-1}}\right)$, where all of the exponents are nonnegative integers. Associated with any vector $c$ in the unit $(n-1)$-cube (and, in particular, with any $b$-point) is an allocation $\left(y^{1}, \ldots, y^{n}\right)$ of the $(n-1)$ commodities among the $n$ traders where for each $1 \leqslant i \leqslant n-1$,

$$
\left(y^{i}\right)_{j}= \begin{cases}1-c_{i} & \text { if } i=j \\ 0 & \text { otherwise }\end{cases}
$$

and

$$
y^{n}=\left(c_{1}, \ldots, c_{n-1}\right) .
$$

Associated in turn with any collection $\left\{u_{i}\right\}$ of utility functions is $\left(u_{1}\left(y^{1}\right), \ldots, u_{n}\left(y^{n}\right)\right)$, the utility outcome (attainable point) which arises from $c$ and is achieved by the allocation.

We now define a collection $\left\{w_{i}\right\}$ of utility functions on $I^{n-1}$. For $1 \leqslant i \leqslant$ $n-1$ and $y \in I^{n-1}$, let

$$
w_{i}(y)= \begin{cases}x_{k} & \text { if } y_{i}=1-b^{k} \text { for some integer } 0 \leqslant k \leqslant p \\ 1 & \text { if } y_{i}=1 \\ 0 & \text { otherwise }\end{cases}
$$

Writing $e=(1, \ldots, 1) \in I^{n-1}$, for each $y \in I^{n-1}$, let

$w_{n}(y)=\left\{\begin{aligned} \max \left\{z:\left(w_{1}(e-y), \ldots, w_{n-1}(e-y), z\right) \in A\right\}, & \\ & \text { if this set is nonempty and if each } y_{i} \in\left\{1, b, b^{2}, \ldots, b^{p}, 0\right\}, \\ 0 \quad \text { otherwise. } & \end{aligned}\right.$

(The function $w_{n}$ is nonzero at only finitely many points.) We claim that the attainable set associated with this collection of functions is the union of $A$ with the corner generated by the point $(1, \ldots, 1,0) \in R^{n}$. To verify this, observe that the definition of $w_{n}$ forces the attainable set to be contained in this union. The reverse containment follows from the observation that any corner point $\left(x_{k_{1}}, \ldots, x_{k_{n-1}}, z\right) \in G$ arises from the $b$-point $\left(b^{k_{1}}, \ldots, b^{k_{n-1}}\right)$, and $(1, \ldots, 1,0)$ arises from the $(n-1)$-vector $(0, \ldots, 0)$ which corresponds to the allocation of one unit of commodity $i$ to trader $i(1 \leqslant i \leqslant n-$ 1), and nothing to trader $n$.

For each $i$, let $v_{i}$ be the infimum of all quasiconcave functions greater than or equal to $w_{i}$. Since the intersection of any collection of convex (level) sets is convex, each $v_{i}$ is quasiconcave. It follows from the construction of the functions $\left\{w_{i}\right\}$ that each $v_{i}$ is monotone increasing and upper-semicontinuous. 
Clearly, $\mathbb{Q}\left(v_{1}, \ldots, v_{n}\right)$ contains $\mathbb{Q}\left(w_{1}, \ldots, w_{n}\right)$. Assume that $\left(y^{1}, \ldots, y^{n}\right)$ is an allocation such that $\left(v_{1}\left(y^{1}\right), \ldots, v_{n}\left(y^{n}\right)\right)$ is not in $Q\left(w_{1}, \ldots, w_{n}\right)$. Without loss of generality (since, for $1 \leqslant i \leqslant n-1, w_{i}$ and hence $v_{i}$ depend only on $\left.\left(y^{i}\right)_{i}\right)$, we can then assume that $\left(y^{1}, \ldots, y^{n}\right)$ corresponds to the $(n-1)$-vector $c=\left(1-\left(y^{1}\right)_{1}, \ldots, 1-\left(y^{n-1}\right)_{n-1}\right)$. Furthermore, since each $v_{i}(y)$ (for $1 \leqslant i \leqslant n-1$ ) is a step-function with jumps only at $y_{i}=1-b^{k}$, we can assume that $c$ is a $b$-point. (That is, for any $\left(y^{1}, \ldots, y^{n}\right)$, let the allocation $\left(z^{1}, \ldots, z^{n}\right)$ be defined by

$$
\begin{aligned}
& \left(z^{i}\right)_{j}=0 \quad \text { if } i \neq j ; \\
& \left(z^{i}\right)_{i}= \begin{cases}1-b^{k} & \text { if } 1-b^{k} \leqslant\left(y^{i}\right)_{i}<1-b^{k+1} \text { for } 0 \leqslant k<p, \\
1-b^{p} & \text { if } 1-b^{p} \leqslant\left(y^{i}\right)_{i}\end{cases}
\end{aligned}
$$

for $1 \leqslant i \leqslant n-1$, and $z^{n}=e-\sum_{k=1}^{n-1} z^{k}$. Then $v_{i}\left(z^{i}\right)=v_{i}\left(y^{i}\right)$ for $1 \leqslant i \leqslant$ $n-1$, and $v_{n}\left(z^{n}\right) \geqslant v_{n}\left(y^{n}\right)$ since $z^{n} \geqslant y^{n}$.) Hence, to show that $\mathbb{Q}\left(v_{1}, \ldots, v_{n}\right)=\mathbb{Q}\left(w_{1}, \ldots, w_{n}\right)$, it suffices to show that each $b$-point gives rise to the same attainable point for both collections of utility functions. That is, we wish to show that $v_{n}(y)=w_{n}(y)$ for every $b$-point $y$. We require a lemma of a primarily geometric nature.

LEMMA. If $\left(b^{k_{1}}, \ldots, b^{k_{n-1}}\right)$ lies on or above the convex hull of the points $\left(b^{l_{1,1}}, \ldots, b^{l_{1, n-1}}\right), \ldots,\left(b^{l_{n-1,1}}, \ldots, b^{l_{n-1, n-1}}\right)$, then for some $j,\left(k_{1}, \ldots, k_{n-1}\right)$ $\leqslant\left(l_{j, 1}, \ldots, l_{j, n-1}\right)$.

Proof OF LEMMA. If the indicated point lies on or above the convex hull of the other $(n-1)$ points, then by definition there is some $\left(t_{1}, \ldots, t_{n-1}\right) \geqslant 0$ with $\sum_{i} t_{i}=1$, for which

$$
\begin{aligned}
b^{k_{1}} & \geqslant t_{1} b^{l_{1,1}}+\cdots+t_{n-1} b^{l_{n-1,1}} \\
& \vdots \\
b^{k_{n-1}} & \geqslant t_{1} b^{l_{1, n-1}}+\cdots+t_{n-1} b^{l_{n-1, n-1}} .
\end{aligned}
$$

Let $S_{m}=\left\{q: l_{q, m}<k_{m}\right\}$. Then, noting the integrality of each $k_{i}$ and writing $t(S)=\Sigma_{i \in S} t_{i}$,

$$
b^{k_{m}} \geqslant t\left(S_{m}\right) b^{k_{m}-1} \text { for all } m \text {. }
$$

Hence for each $m, b \geqslant t\left(S_{m}\right)$, and adding these inequalities yields

$$
1 \geqslant(n-1) b>\sum_{m} t\left(S_{m}\right) \text {. }
$$

Since $\Sigma_{i} t_{i}=1$, it must be that some $t_{j}$ never appears on the right-hand side of the inequality. For this $j$, the conclusion of the lemma holds.

Let $y$ be any $b$-point. Then $v_{n}(y)>w_{n}(y)$ only if $y$ lies on or above the convex hull of a collection of $b$-points $\left\{y^{i}\right\}$, each of which satisfies

$$
w_{n}\left(y^{i}\right)>w_{n}(y) .
$$

However, we can assume, by Carathéodory's Theorem, that the collection 
$\left\{y^{i}\right\}$ consists of $(n-1)$ points. The lemma then implies that, for some specific $i, y \geqslant y^{i}$, and the monotonicity of $w_{n}$ contradicts (*). Therefore $v_{n}(y)=w_{n}(y)$ for all $b$-points $y$, and this completes the proof that $\mathbb{Q}\left(v_{1}, \ldots, v_{n}\right)=\mathbb{Q}\left(w_{1}, \ldots, w_{n}\right)$.

We next modify the functions $\left\{v_{i}\right\}$, yielding utility functions $\left\{u_{i}\right\}$ which are continuous as well as monotone increasing and quasiconcave.

Select any $0<\varepsilon<\frac{1}{2}\left((n-1)^{-1}-b\right) b^{p-1}$. For each $1 \leqslant i \leqslant n$ and $y \in$ $I^{n-1}$, define

$$
\begin{array}{r}
u_{i}(y)=\min \left\{h: v_{i}(t y+(1-t) e) \leqslant t h+(1-t)(1+1 / \varepsilon)\right. \\
\text { for all } 0 \leqslant t \leqslant 1\} .
\end{array}
$$

This construction attaches "inclines" to the graph of each $v_{i}$ along each line of discontinuity, in such a manner that each incline has a width (at its base) no greater than $\varepsilon$. (The inclines arise figuratively as the boundaries of the shadow formed when a light, at a height of $1+1 / \varepsilon$ above the point $e=(1, \ldots, 1)$, shines down on the graph of $v_{i}$.) Each $u_{i}$ is monotonic.

To verify that each $u_{i}$ is continuous, consider an allocation $y$ of commodities to trader $i$. Assume for the moment that $\min \left(1-y_{j}\right)=\alpha>0$. For any $0<\delta<\alpha / 2$, consider any $z$ for which $\left|z_{j}-y_{j}\right| \leqslant \delta$ for all $j$. Let $\gamma=1-$ $\delta / \alpha$. Then $0<\gamma<1$ and $z \leqslant \gamma y+(1-\gamma) e$. Therefore,

$$
u_{i}(z) \leqslant u_{i}(\gamma y+(1-\gamma) e) \leqslant \gamma u_{i}(y)+(1-\gamma)(1+1 / \varepsilon),
$$

and

$$
u_{i}(z)-u_{i}(y) \leqslant(\delta / \alpha)(1+1 / \varepsilon) .
$$

Similarly, since $\min \left(1-z_{j}\right)>\alpha / 2$, we have

$$
u_{i}(y)-u_{i}(z)<(2 \delta / \alpha)(1+1 / \varepsilon) \text {. }
$$

Therefore, if $z$ is near $y$ ( $\delta$ is small), then $u_{i}(z)$ is near $u_{i}(y)$. We need to also treat the case in which $y_{j}=1$ for some $j$. However, $w_{i}$ was originally defined so that $u_{i}\left(y_{1}, \ldots, y_{j-1}, \bar{y}, y_{j+1}, \ldots, y_{n-1}\right)$ is constant for all $1-b^{p} \leqslant \bar{y} \leqslant 1$ (if $1 \leqslant i \leqslant n-1$ ), or for all $b \leqslant \bar{y} \leqslant 1$ (if $i=n$; this is why we took $x_{0}=x_{1}=0$ ). Hence, the continuity of $u_{i}$ at $y$ follows as before.

It is possible that $u_{n}$ is not quasiconcave. If this is the case, let $\tilde{u}_{n}$ be the function defined above, and redefine $u_{n}$ as the infimum of all quasiconcave functions greater than or equal to $\tilde{u}_{n}$. (This operation, applied to a continuous function over a polyhedral domain, yields a continuous function; see [11]. It also preserves monotonicity.)

It remains only to show that $\mathbb{Q}\left(u_{1}, \ldots, u_{n}\right)=\mathbb{Q}\left(v_{1}, \ldots, v_{n}\right)$. Clearly, $\mathbb{Q}\left(v_{1}, \ldots, v_{n}\right)$ is contained in $\mathbb{Q}\left(u_{1}, \ldots, u_{n}\right)$. Consider any allocation $\left(y^{1}, \ldots, y^{n}\right)$. We wish to show that $\left(u_{1}\left(y^{1}\right), \ldots, u_{n}\left(y^{n}\right)\right) \in \mathbb{Q}\left(v_{1}, \ldots, v_{n}\right)$. Let $\left(z^{1}, \ldots, z^{n}\right)$ be the allocation defined by

$$
\left(z^{i}\right)_{j}=0 \text { if } i \neq j
$$




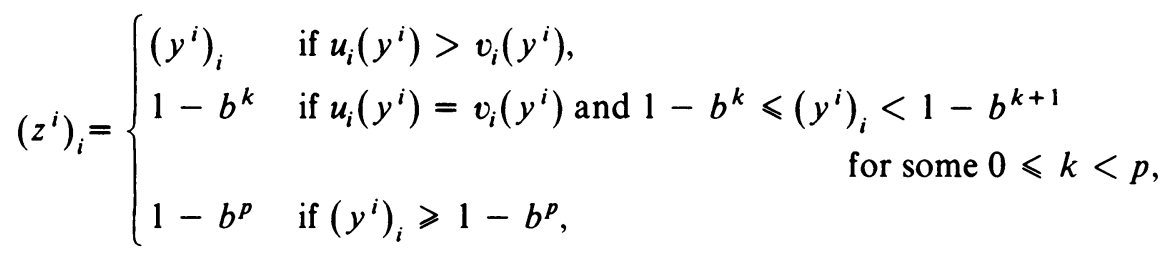

for $1 \leqslant i \leqslant n-1$, and $z^{n}=e-\sum_{k=1}^{n-1} z^{k}$. Then, for each $1 \leqslant i \leqslant n-1$, there is an integer $0 \leqslant k_{i} \leqslant p$ such that $1-b^{k_{i}}-\varepsilon<\left(z^{i}\right)_{i} \leqslant 1-b^{k_{i}}$ and $b^{k_{i}} \leqslant\left(z^{n}\right)_{i}<b^{k_{i}}+\varepsilon$. Also, $u_{i}\left(z^{i}\right)=u_{i}\left(y^{i}\right)$ for $1 \leqslant i \leqslant n-1$, and $u_{n}\left(z^{n}\right) \geqslant$ $u_{n}\left(y^{n}\right)$ since $z^{n} \geqslant y^{n}$. Hence, it will suffice to show that $\left(u_{1}\left(z^{1}\right), \ldots, u_{n}\left(z^{n}\right)\right)$ $\in \mathbb{Q}\left(v_{1}, \ldots, v_{n}\right)$.

Define $\left(\bar{z}^{1}, \ldots, \bar{z}^{n}\right)$ by

$$
\left(\bar{z}^{i}\right)_{j}=0 \quad \text { if } i \neq j ; \quad\left(\bar{z}^{i}\right)_{i}=1-b^{k_{i}}, \quad \text { for } 1 \leqslant i \leqslant n-1,
$$

and $\bar{z}^{n}=e-\sum_{k=1}^{n-1} \bar{z}^{k}$. We will show that $u_{i}\left(z^{i}\right) \leqslant v_{i}\left(\bar{z}^{i}\right)$ for all $i$. If $1 \leqslant i \leqslant$ $n-1, z^{i} \leqslant \bar{z}^{i}$ and $u_{i}\left(z^{i}\right) \leqslant u_{i}\left(\bar{z}^{i}\right)=v_{i}\left(\bar{z}^{i}\right)$. Therefore, it only remains to show that $u_{n}\left(z^{n}\right)=v_{n}\left(\bar{z}^{n}\right)$. We require an extension of the previous lemma.

LEMMA. If $\left(b^{k_{1}}+2 \varepsilon, \ldots, b^{k_{n-1}}+2 \varepsilon\right)$ lies on or above the convex hull of the points $\left(b^{l_{1,1}}, \ldots, b^{l_{1, n-1}}\right), \ldots,\left(b^{l_{n-1,1}}, \ldots, b^{l_{n-1, n-1}}\right)$, then for some $i$, $\left(k_{1}, \ldots, k_{n-1}\right) \leqslant\left(l_{i, 1}, \ldots, l_{i, n-1}\right)$.

Proof OF LEMMA. Proceeding as in the proof of the previous lemma, we eventually find that for every $j, b^{k_{j}}+2 \varepsilon \geqslant t\left(S_{j}\right) b^{k_{j}-1}$ and, therefore,

$$
b+2 \varepsilon b^{i-k_{j}} \geqslant t\left(S_{j}\right) \text {. }
$$

Summing,

$$
(n-1) b+2 \varepsilon \sum_{j} b^{1-k_{j}} \geqslant \sum_{j} t\left(S_{j}\right) .
$$

However, the choice of $\varepsilon$ implies that

$$
1>(n-1) b+2 \varepsilon(n-1) b^{1-p},
$$

and since all $k_{j} \leqslant p$, this in turn implies that some index $i$ does not appear in any $S_{j}$. Therefore, the point $\left(b^{k_{1}}, \ldots, b^{k_{n-1}}\right)$ is greater than or equal to the point $\left(b^{l_{i, 1}}, \ldots, b^{l_{i, n-1}}\right)$ in all components.

We first show that $\tilde{u}_{n}\left(z^{n}\right)=v_{n}\left(\bar{z}^{n}\right)$. If $\bar{z}+2 \varepsilon e$ is on or above the convex hull of a collection of $b$-points, the lemma asserts that $\bar{z}^{n}$ lies above one of the points. Hence, $v_{n}\left(\bar{z}^{n}+2 \varepsilon e\right)=v_{n}\left(\bar{z}^{n}\right)$. Since $\tilde{u}_{n}$ differs from $v_{n}$ only at points within $\varepsilon$ (in every component) of a discontinuity of $v_{n}$,

$$
\tilde{u}_{n}\left(\bar{z}^{n}+\varepsilon e\right)=v_{n}\left(\bar{z}^{n}+\varepsilon e\right)=v_{n}\left(\bar{z}^{n}\right) \text {. }
$$

Also, since $\bar{z}^{n}+\varepsilon e \geqslant z^{n} \geqslant \bar{z}^{n}$, the monotonicity of the functions under consideration allows us to conclude that

$$
v_{n}\left(\bar{z}^{n}\right)=\tilde{u}_{n}\left(\bar{z}^{n}+\varepsilon e\right) \geqslant \tilde{u}_{n}\left(z^{n}\right) \geqslant \tilde{u}_{n}\left(\bar{z}^{n}\right) \geqslant \tilde{v}_{n}\left(\bar{z}^{n}\right),
$$

which yields the desired result.

Finally, we show that $u_{n}\left(z^{n}\right)=\tilde{u}_{n}\left(z^{n}\right)$. Assume that this is false. Then $z^{n}$ is 
on or above a convex combination of $(n-1)$-vectors $c^{k}$, where for each $k$, $\tilde{u}_{n}\left(c^{k}\right)>\tilde{u}_{n}\left(z^{n}\right)$. Furthermore, it can be assumed without loss of generality that each $c^{k}$ is no more than $\varepsilon e$ below a convex combination of $b$-points $b^{(k, l)}$, where for each $l, \tilde{u}_{n}\left(b^{(k, l)}\right)>\tilde{u}_{n}\left(z^{n}\right)$. Therefore, $z^{n}+\varepsilon e$ lies on or above a convex combination of points in the collection $\left\{b^{(k, l)}\right\}$. Since $\bar{z}^{n}+2 \varepsilon e \geqslant z^{n}$ $+\varepsilon e>z^{n} \geqslant \bar{z}^{n}$, the lemma then guarantees that $\bar{z}^{n}$ lies above one of these $b$-points $b^{\left(k_{0} l_{0}\right)}$. It follows that

$$
\tilde{u}_{n}\left(z^{n}\right) \geqslant \tilde{u}_{n}\left(\bar{z}^{n}\right) \geqslant \tilde{u}_{n}\left(b^{\left(k_{0}, l_{0}\right)}\right)>\tilde{u}_{n}\left(z^{n}\right)
$$

a contradiction. Therefore, $u_{n}\left(z^{n}\right)=\tilde{u}_{n}\left(z^{n}\right)=v_{n}\left(\bar{z}^{n}\right)$. This completes the proof that $\mathbb{Q}\left(u_{1}, \ldots, u_{n}\right)=\mathbb{Q}\left(v_{1}, \ldots, v_{n}\right)$.

Thus we have constructed a collection $\left\{u_{i}\right\}$ of utility functions which are continuous, monotone increasing, and quasiconcave as desired. The attainable set of this collection is the union of $A$ with the corner generated by $(1, \ldots, 1,0)$. In order to eliminate this extraneous corner, we "intersect" this market with $(n-1)$ others, each constructed in a similar manner but distinguishing traders $1,2, \ldots,(n-1)$ in turn rather than trader $n$. (See [2] for details on the intersection of markets. All that need be observed here is that the procedure of intersection involves taking the minimum of finite collections of utility functions, a process which preserves the properties of continuity, monotonicity, and quasiconcavity.) This completes our proof of the theorem.

It should be noted that, despite the rather tedious notational details of the preceding proof, the construction is actually quite simple. It merely involves an exponential scaling transformation of the corner points, designed to make them extreme points of a comprehensive set with convex cross-sections perpendicular to the $n$th coordinate axis. Unfortunately, this idea does not generalize directly to sets more complicated than unions of corners, nor does it succeed in the limit as the number of corner points is allowed to become infinite. Hence, the question of completely characterizing those attainable sets arising from utility functions of the type considered here remains open.

A second point to be noted concerns the "complexity" of the market constructed to generate the desired attainable set. In general, our construction requires the use of $n(n-1)$ commodities, most of which are introduced at the very end solely to eliminate one extraneous point. An analysis of minimal constructions, in the direction of [7], would be of interest.

\section{BIBLIOGRAPHY}

1. L. J. Billera, On games without side payments arising from a general class of markets, J. Math. Econom. 1 (1974), 129-139.

2. L. J. Billera and R. E. Bixby, A characterization of Pareto surfaces, Proc. Amer. Math. Soc. 41 (1973), 261-267. MR 48 \#3512.

3. _ A characterization of polyhedral market games, Internat. J. Game Theory 2 (1973), 253-261. MR 51 \# 9845.

4. , Market representations of n-person games, Bull. Amer. Math. Soc. 80 (1974), $522-526$. 
5. __ Pareto surfaces of complexity 1, SIAM J. Appl. Math. 30 (1976), 81-89.

6. E. Kalai, On game-type subsets, Internat. J. Game Theory 4 (1975), 141-150.

7. E. Kalai and M. Smorodinsky, On a game theoretic notion of complexity for compact convex sets, Proc. Amer. Math. Soc. 49 (1975), 416-420. MR 51 \# 4948.

8. A. Mas-Colell, $A$ further result on the representation of games by markets, J. Econom. Theory 10 (1975), 117-122.

9. H. E. Scarf, The core of an N-person game, Econometrica 35 (1967), 50-69. MR 38 \#3051.

10. L. S. Shapley and M. Shubik, On market games, J. Econom. Theory 1 (1969), 9-25.

11. R. J. Weber, Quasiconcavification and continuity, working paper.

Department of Mathematics, University of Connecticut, Storrs, Connecticut 06268 